\title{
Erratum to: Lesinurad, a novel, oral compound for gout, acts to decrease serum uric acid through inhibition of urate transporters in the kidney
}

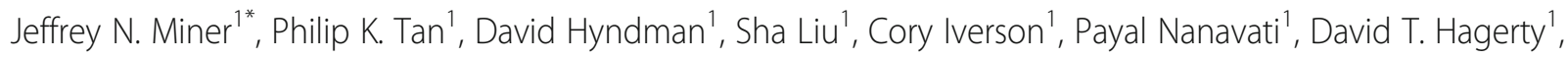
Kimberly Manhard', Zancong Shen ${ }^{1}$, Jean-Luc Girardet ${ }^{1}$, Li-Tain Yeh', Robert Terkeltaub² and Barry Quart ${ }^{1}$

\section{Erratum}

Unfortunately, after publication of this article [1], it was noticed that the name of Jeffrey N. Miner was captured incorrectly during the production process. The middle initial ' $\mathrm{N}$ ' was missing from the list. The corrected author list can be seen above and the original article has been updated to correct this error.

\footnotetext{
Author details

'Ardea Biosciences, Inc., 9390 Towne Centre Drive, San Diego, CA 92121, USA. ${ }^{2}$ University of California San Diego, 9500 Gilman Dr, La Jolla, CA 92093 USA.
}

Received: 7 October 2016 Accepted: 7 October 2016

Published online: 12 October 2016

\section{Reference}

1. Miner JN, Tan PK, Hyndman D, Liu S, Iverson C, Nanavati P, Hagerty DT, Manhard K, Shen Z, Girardet J-L, Yeh L-T, Terkeltaub R, Quart B. Lesinurad, a novel, oral compound for gout, acts to decrease serum uric acid through inhibition of urate transporters in the kidney. Arthritis Res Ther. 2016;18:214. doi:10.1186/s13075-016-1107-x.

* Correspondence: jminer@ardeabio.com

'Ardea Biosciences, Inc., 9390 Towne Centre Drive, San Diego, CA 92121, USA

Full list of author information is available at the end of the article 